\title{
On Vietoris Soft Topology I
}

\author{
Q. R. Shakir* \\ School of Mathematics, Statistics and Applied Mathematics, National University of Ireland- \\ Galway, Galway, Ireland
}

Received 20 May 2015, accepted in final revised form 19 September 2015

\begin{abstract}
In this article, we define the hyperspace of soft closed sets of a soft topological space $\left(F_{A}, \tau\right)$. In addition, we define the Vietoris soft topology, $\tilde{\tau}_{v}$, by determining the soft base of this topology which has the form $\left\langle\left(F_{H_{1},}, F_{H_{2}, \ldots,} F_{H_{n}}\right\rangle\right.$, where $F_{H_{1},} F_{H_{2}, \ldots,} F_{H_{n}}$ are soft open sets in $\left(F_{A}, \tau\right)$. Some properties of this topology are also investigated. The impact of introducing the Vietoris soft topology is to enable us to understand many properties of the structure of soft topologies corresponding to it.

Keywords: Soft set; Soft open set; Soft hyperspace; Vietoris soft topology.

() 2016 JSR Publications. ISSN: 2070-0237 (Print); 2070-0245 (Online). All rights reserved. doi: http://dx.doi.org/10.3329/jsr.v8i1.23440

J. Sci. Res. 8 (1), 13-19 (2016)
\end{abstract}

\section{Introduction}

The comparison between the number of the articles, researches and applications of the theory of soft set and its young age which introduced in 1999 by Molodtsov [1] reveals the significance of such a mathematical tool for dealing with uncertainty and vagueness. The appearance of this theory was welcomed by mathematicians by investigating potential properties as well as introducing and generalising many concepts that certain complicated problems can be solved by building models depending on.

Shabir [2] introduced the soft topological spaces which are defined over an initial universe with a fixed set of parameters and studied some basic notions of soft topological spaces such as soft open, soft closed sets, soft subspace, soft closure, soft neighbourhood of a point and soft separation axioms. This work followed by many researches that dealt with various generalizations of this certain types of open sets.

${ }^{*}$ Corresponding author: Q.shakir2@nuigalway.ie 
There is a comprehensive survey covers the development of soft set theory and its applications as reported elsewhere [3]. The literature of studying hyperspaces in topological space has a rich history which started almost ten decades ago when Vietoris [4] introduced one hyperspace called finite or the Vietoris hyperspace. After that many other hyperspaces were introduced and investigated their structures. The significance of hyperspaces theory is represented by investigating their properties which can help to understand the structures of topological spaces corresponding to them. Many types of hyperspaces were investigated by E. Michael [5].

This article adds a new type of hyperspaces completely depends on soft open and soft closed sets in a soft topological space. We recall the definition of the Vietoris hyperspace by using both open and closed soft sets. Then we investigate some properties of this soft hyperspace. All definitions which stated are defined over an initial universe with a fixed set of parameters.

\section{Preliminaries}

Let $U$ be an initial universe set and $E$ be the set of all possible parameters with respect to $\boldsymbol{U}$ and $\mathcal{P}(\boldsymbol{U})$ represents the power set of $\boldsymbol{U}$. The parameters are often attributes or properties of the objects in the initial universe.

Definition 2.1: [1] A soft set $\boldsymbol{F}_{A}$ on the universe set $\boldsymbol{U}$ is denoted by the set of ordered pairs:

$F_{A}=\left\{\left(x, f_{A}(x)\right): x \in E, f_{A}(x) \in \mathcal{P}(U)\right\}$, where $f_{A}: E \rightarrow \mathcal{P}(U)$ such that $f_{A}(x)=\emptyset$ if $x \notin A$. $f_{A}$ is called an approximate function of the soft set $\boldsymbol{F}_{A}$. The value of $\boldsymbol{f}_{A}$ may be an arbitrary.

Example 2.2: Suppose that there are eight cars in the universe $U=\left\{c_{1}, c_{2}, \ldots, c_{8}\right\}$ and let $\boldsymbol{E}=\left\{\boldsymbol{x}_{1}, \boldsymbol{x}_{2}, \boldsymbol{x}_{3}, \boldsymbol{x}_{4}, \boldsymbol{x}_{5}\right\}$ is the set of decision parameters such that $\boldsymbol{x}_{1}=$ new, $\boldsymbol{x}_{2}=$ expensive, $\quad x_{3}=$ high-tech, $x_{4}=$ model, $x_{5}=$ interior design. Consider the map $\boldsymbol{f}_{A} \equiv$ cars(attributes), so for example $\boldsymbol{f}_{A}\left(\boldsymbol{x}_{3}\right)$ means "cars(high-tech)". Thus the functional value of $\boldsymbol{f}_{A}\left(\boldsymbol{x}_{3}\right)$ is the set $\left\{\boldsymbol{c} \in U_{:} \boldsymbol{c}\right.$ is high-tech $\}$. Now let $\boldsymbol{A}=\left\{\boldsymbol{x}_{2}, \boldsymbol{x}_{3}, \boldsymbol{x}_{5}\right\}$ and $f_{A}\left(x_{2}\right)=\left\{c_{2}, c_{6}\right\}, f_{A}\left(x_{3}\right)=\left\{c_{1}, c_{3}, c_{4}\right\}, \quad f_{A}\left(x_{5}\right)=\left\{c_{1}, c_{7}, c_{8}\right\}$. Then the soft set $F_{A}=\left\{\left(x_{2},\left\{c_{2}, c_{6}\right\}\right),\left(x_{3},\left\{c_{1}, c_{3}, c_{4}\right\}\right),\left(x_{5},\left\{c_{1}, c_{7}, c_{8}\right\}\right)\right\}$.

Definition 2.3: [1] Let $F_{A}$ be a soft set, if $\boldsymbol{f}_{A}(\boldsymbol{x})=U$ for all $\boldsymbol{x} \in A$, then $\boldsymbol{F}_{A}$ is called an A-universe soft set and is denoted by $\boldsymbol{F}_{\Re}$.

If $\boldsymbol{A}=\boldsymbol{E}$, then $\boldsymbol{F}_{\mathrm{B}}$ is called a universe soft set.

Example 2.4: Let $U=\left\{u_{1}, u_{2}, u_{3}\right\}, E=\left\{x_{1}, x_{2}, x_{3}\right\}$ and $A=\left\{x_{1}, x_{2}\right\}$, then $F_{\mathscr{A}}=\left\{\left(x_{1}, U\right)\right.$, $\left.\left(x_{2}, U\right)\right\}$ and $F_{\mathscr{Z}}=\left\{\left(x_{1}, U\right),\left(x_{2}, U\right),\left(x_{3}, U\right)\right\}$.

Definition 2.5: [1] Let $\boldsymbol{F}_{A}$ be a soft set, if $\boldsymbol{f}_{A}(\boldsymbol{x})=\emptyset$ for all $\boldsymbol{x} \in \boldsymbol{E}$, then $\boldsymbol{F}_{A}$ is called the empty soft set and denoted by $\boldsymbol{F}_{\boldsymbol{\Phi}}$. 
Example 2.6: Let $U=\left\{u_{1}, u_{2}, u_{3}, u_{4}, u_{5}\right\}$ and $E=\left\{x_{1}, x_{2}, x_{3}\right\}$, then $F_{\Phi}=\left\{\left(x_{1}, \emptyset\right)\right.$, $\left.\left(x_{2}, \emptyset\right),\left(x_{3}, \emptyset\right)\right\}$.

Definition 2.7: [1] Let $F_{A}$ and $F_{B}$ be soft sets. Then $F_{A}$ is a soft subset of $\boldsymbol{F}_{B}$ if $f_{A}(x) \subseteq f_{B}(x)$ for all $x \in E$ and is denoted by $F_{A} \subseteq F_{B}$.

Example 2.8: Let $U=\left\{u_{1}, u_{2}, u_{3}, u_{4}, u_{5}\right\}, E=\left\{x_{1}, x_{2}, x_{3}, x_{4}, x_{5}\right\}, A=\left\{x_{1}, x_{4}\right\}$ and $B=\left\{x_{4}\right\}$. Suppose $F_{A}=\left\{\left(x_{1},\left\{u_{1}, u_{5}\right)\right\},\left(x_{4},\left\{u_{2}, u_{3}, u_{4}\right\}\right)\right\}$ and $F_{B}=\left\{\left(x_{4},\left\{u_{2}, u_{3}\right\}\right)\right\}$, then $F_{B} \subseteq F_{A}$.

Definition 2.9: [1] Let $F_{A}$ and $F_{B}$ be soft sets, then $F_{A}$ and $F_{B}$ are soft equal if $f_{A}(x)=f_{B}(x)$ for all $x \in E$.

Definition 2.10: [1] Let $\boldsymbol{F}_{A}$ and $\boldsymbol{F}_{B}$ be soft sets, then the union of $\boldsymbol{F}_{A}$ and $\boldsymbol{F}_{B}$ (denoted by $\left.F_{A} \square F_{B}\right)$ is defined by $F_{A} \square F_{B}=f_{A}(x) \cup f_{B}(x)$ for all $\boldsymbol{x} \in A \cup B$.

Example 2.11: Let $U=\left\{u_{1}, u_{2}, u_{3}\right\}, E=\left\{x_{1}, x_{2}, x_{3}\right\}, A=\left\{x_{1}, x_{2}\right\}, B=\left\{x_{3}\right\}$,

$F_{A}=\left\{\left(x_{1},\left\{u_{1}, u_{2}\right\}\right),\left(x_{2},\left\{u_{2}\right\}\right)\right\}$ and $F_{B}=\left\{\left(x_{2},\left\{u_{1}, u_{3}\right\}\right),\left(x_{3},\left\{u_{3}\right\}\right)\right\}$. Then

$F_{A} \cup F_{B}=\left\{\left(x_{1},\left\{u_{1}, u_{2}\right\}\right),\left(x_{2}, U\right),\left(x_{3},\left\{u_{3}\right\}\right)\right\}$.

Definition 2.12: [1] Let $\boldsymbol{F}_{A}$ and $\boldsymbol{F}_{B}$ be soft sets, then the intersection of $\boldsymbol{F}_{A}$ and $\boldsymbol{F}_{B}$ (denoted by $\boldsymbol{F}_{A} \widetilde{F}_{\boldsymbol{B}}$ ) is defined by $\boldsymbol{F}_{A} \widetilde{F}_{F_{B}}=\boldsymbol{f}_{A}(\boldsymbol{x}) \cap \boldsymbol{f}_{B}(\boldsymbol{x})$ for all $\boldsymbol{x} \in A \cap \boldsymbol{B}$.

Example 2.13: Let $U=\left\{u_{1}, u_{2}, u_{3}, u_{4}\right\}, E=\left\{x_{1}, x_{2}, x_{3}, x_{4}\right\}, A=\left\{x_{1}, x_{4}\right\}, B=\left\{x_{1}, x_{3}\right\}$, $F_{A}=\left\{\left(x_{1},\left\{u_{1}\right\}\right),\left(x_{4},\left\{u_{2}, u_{3}, u_{4}\right\}\right)\right\} \quad$ and $F_{B}=\left\{\left(x_{1},\left\{u_{1}, u_{3}\right\}\right),\left(x_{3},\left\{u_{3}\right\}\right)\right\}$. Then $F_{A} \widetilde{F_{B}}=\left\{\left(x_{1},\left\{u_{1}\right\}\right)\right\}$.

Definition 2.14: [1] Let $\boldsymbol{F}_{A}$ be a soft set, $\boldsymbol{\alpha}=(\boldsymbol{x},\{u\})$ is a nonempty soft element of $\boldsymbol{F}_{A}$, denoted by $\alpha \tilde{\boldsymbol{E}} \mathbf{F}_{A}$ if there exists $\boldsymbol{x} \in \boldsymbol{E}$ and $\mathbf{u} \in \boldsymbol{f}_{A}(\boldsymbol{x})$.

Notice that the singleton set of a soft point is denoted by $\boldsymbol{F}_{\alpha}$.

Example 2.15: Let $U=\left\{u_{1}, u_{2}, u_{3}\right\}, E=\left\{x_{1}, x_{2}, x_{3}\right\}, A=\left\{x_{2}, x_{3}\right\}$ and let $F_{A}=\left\{\left(x_{2},\left\{u_{2}, u_{3}\right\}\right),\left(x_{3},\left\{u_{1}, u_{2}\right\}\right)\right\}$, then the following are nonempty elements in $F_{A}$ :

$\alpha_{1}=\left(x_{2},\left\{u_{2}\right\}\right) \tilde{\epsilon} F_{A} ;$ since $u_{2} \in f_{A}\left(x_{2}\right)=\left\{u_{2}, u_{3}\right\}$.

$\alpha_{2}=\left(x_{2},\left\{u_{3}\right\}\right) \tilde{E} F_{A} ;$ since $u_{3} \in f_{A}\left(x_{2}\right)=\left\{u_{2}, u_{3}\right\}$.

$\alpha_{3}=\left(x_{3},\left\{u_{1}\right\}\right) \tilde{E} F_{A} ;$ since $u_{1} \in f_{A}\left(x_{3}\right)=\left\{u_{1}, u_{2}\right\}$.

$\alpha_{4}=\left(x_{3},\left\{u_{2}\right\}\right) \tilde{\epsilon} F_{A} ;$ since $u_{2} \in f_{A}\left(x_{3}\right)=\left\{u_{1}, u_{2}\right\}$.

Definition 2.16: [1] Let $\mathbf{F}_{\mathrm{A}}$ be a soft set, the soft complement of $\mathbf{F}_{\mathrm{A}}$ (denoted by $\mathbf{F}_{A}^{\tau}$ ) is defined by the approximate function $\mathbf{F}_{A}^{\prec}=\mathbf{f}_{A}^{f}(\boldsymbol{x})$, where $\mathbf{f}_{A}^{f}(\boldsymbol{x})=U-\mathbf{f}_{A}(\boldsymbol{x})$ for all $\boldsymbol{x} \in A$.

Example 2.17: Let $U=\left\{u_{1}, u_{2,}, u_{3}\right\}, \quad E=\left\{x_{1}, x_{2}, x_{3}\right\}, \quad A=\left\{x_{2}, x_{3}\right\} \quad$ and let $F_{A}=\left\{\left(x_{1},\left\{u_{2}\right\}\right),\left(x_{3},\left\{u_{1}, u_{3}\right\}\right)\right\}$. Then $\mathrm{F}_{A}^{\ell}=\left\{\left(x_{1},\left\{u_{1}, u_{3}\right\}\right),\left(x_{3},\left\{u_{2}\right\}\right)\right\}$. 
Definition 2.18: [2] Let $\boldsymbol{F}_{A}$ be a soft set, a soft topology on $\boldsymbol{F}_{A}$, denoted by $\tilde{\boldsymbol{\tau}}$, is a collection of soft subsets of $\boldsymbol{F}_{\boldsymbol{A}}$ satisfying the following conditions:

(1) $F_{\Phi_{s}} F_{A} \in \tilde{\tau}$.

(2) If $\left\{F_{E_{i}} \subseteq F_{A}: i \in I \subseteq \mathbb{N}\right\} \subseteq \tilde{\tau}$, then $\widetilde{\sigma_{i \in I}}, F_{H_{i}} \in \tilde{\tau}$.

(3) If $\left\{F_{E_{t}} \subseteq F_{A}: 1 \leq i \leq n, n \in \mathbb{N}\right\} \subseteq \tilde{\tau}$, then $\widetilde{n_{t=1}^{n}} F_{H_{t}} \in \tilde{\tau}$.

Then $\tilde{\boldsymbol{\tau}}$ is called a soft topology and the pair $\left(\boldsymbol{F}_{A}, \tilde{\boldsymbol{\tau}}\right)$ is called a soft topological space.

Example 2.19: Let $U=\left\{u_{1}, u_{2}, u_{3}\right\}, \quad E=\left\{x_{1}, x_{2}, x_{3}\right\}, \quad A=\left\{x_{1}, x_{2}\right\}, \quad$ then $\left(F_{A}, \tilde{\tau}\right)=\left\{F_{\Phi}, F_{A_{1}}, F_{A_{2}}, F_{A_{3}}, F_{A}\right\}$ is a soft topological space, where $F_{A_{1}}=\left\{\left(x_{1},\left\{u_{2}\right\}\right)\right\}$, $F_{A_{2}}=\left\{\left(x_{1},\left\{u_{2}\right\}\right),\left(x_{2},\left\{u_{2}\right\}\right)\right\}, F_{A_{3}}=\left\{\left(x_{1},\left\{u_{1}, u_{2}\right\}\right),\left(x_{2},\left\{u_{2}\right\}\right)\right\}$.

Definition 2.20: [2] Let $\left(\boldsymbol{F}_{A}, \tilde{\boldsymbol{\tau}}\right)$ be a soft topological space and $\boldsymbol{F}_{B} \subseteq \boldsymbol{F}_{A}$. Then $\boldsymbol{F}_{B}$ is said to be a soft closed if $\boldsymbol{F}_{B}^{t}$ is a soft open set.

Example 2.21: Consider the previous example, then $F_{B}=\left\{\left(x_{1},\left\{u_{1}, u_{3}\right\}\right),\left(x_{2},\left\{u_{1}, u_{3}\right\}\right)\right\}$ is a soft closed set.

Definition 2.22: [2] Let $\left(F_{A}, \tilde{\tau}\right)$ be a soft topological space and $\boldsymbol{\alpha} \tilde{\boldsymbol{\epsilon}} \boldsymbol{F}_{A}$, if there is a soft open set $\boldsymbol{F}_{B}$ such that $\boldsymbol{\alpha} \tilde{\boldsymbol{\epsilon}} \boldsymbol{F}_{B}$ then $\boldsymbol{F}_{B}$ is called a soft open neighbourhood ( or soft neighbourhood ) of $\boldsymbol{\alpha}$.

The set of all neighbourhood of $\boldsymbol{\alpha}$ is called the family of soft neighbourhood of $\boldsymbol{\alpha}$ and denoted by $\boldsymbol{V}(\boldsymbol{\alpha})=\left\{F_{B}: F_{B} \in \tilde{\tau}\right.$ and $\left.\boldsymbol{\alpha} \tilde{\boldsymbol{E}} F_{B}\right\}$.

Definition 2.23: A soft point is called a soft isolated point $\boldsymbol{\alpha}$ if and only if $\boldsymbol{F}_{\boldsymbol{\alpha}}$ is soft open.

Definition 2.24: [6] Let $\left(\boldsymbol{F}_{A}, \tilde{\tau}\right)$ be a soft topological space. Then a family $\tilde{\boldsymbol{\beta}} \subseteq \tilde{\boldsymbol{\tau}}$ is called a soft base for $\left(F_{A}, \tilde{\tau}\right)$ if for every soft open set $F_{H} \neq F_{\phi}$, there exist $F_{B_{t}} \in \tilde{\beta}$, $i \in I$, such that $F_{H}=\mathbb{Q} F_{B_{i}} ; i \in I$.

Definition 2.25: [2] A soft topological space $\left(\boldsymbol{F}_{A}, \tilde{\tau}\right)$ is called $\tilde{T}_{0}$ if for each $\alpha_{1}, \alpha_{2} \tilde{\boldsymbol{\epsilon}} F_{A}$ with $\alpha_{1} \neq \alpha_{2}$ there exist soft open sets $F_{H_{1}} \in \mathcal{V}\left(\alpha_{1}\right)$ and $F_{H_{2}} \in \mathcal{V}\left(\alpha_{2}\right)$ such that $\alpha_{2} \notin F_{H_{1}}$ or $\alpha_{1} \notin F_{H_{z}}$.

Definition 2.26: [2] A soft topological space $\left(F_{A}, \tilde{\tau}\right)$ is called $\widetilde{T}_{1}$ if for each $\alpha_{1}, \alpha_{2} \tilde{\boldsymbol{E}} F_{A}$ with $\alpha_{1} \neq \alpha_{2}$ there exist soft open sets $F_{H_{1}} \in \mathcal{V}\left(\alpha_{1}\right)$ and $F_{H_{2}} \in \mathcal{V}\left(\alpha_{2}\right)$ such that $\alpha_{2} \notin F_{H_{1}}$ and $\alpha_{1} \notin F_{H_{2}}$.

Definition 2.27: A sequence of soft sets $\left\{F_{H_{n}}\right\}_{n=1}^{m \infty}$ is eventually contained in a soft set $\mathrm{F}_{K}$ if there is an integer $N \in \mathbb{N}$ such that $F_{H_{n}} \stackrel{\varrho}{\subseteq} F_{K}$ for each $n \geq N$. 
If a sequence $\left\{F_{H_{n}}\right\}_{n=1}^{\mathrm{w}}$ of soft sets in a soft topological space contained eventually in each soft neighbourhood of a soft set $\boldsymbol{F}_{K}$ then this sequence converges to $\boldsymbol{F}_{K}$, this soft convergence is denoted by $F_{H_{n}} \approx F_{K}$.

Definition 2.28: A sequence $\left\{\boldsymbol{F}_{H_{n}}\right\}_{n=1}^{=\infty}$ is called an increasing sequence if $\boldsymbol{F}_{H_{n}} \subseteq \boldsymbol{F}_{H_{n+1}}$ for each $n \in \mathbb{N}$.

\section{The Vietoris soft hyperspace}

Definition 3.1: The soft hyperspace of the soft topological space $\boldsymbol{F}_{\boldsymbol{A}}$ is the following collection $\overline{C L}\left(F_{A}\right)=\left\{F_{K} \subseteq F_{A}: F_{K}\right.$ is a soft closed in $\left.F_{A}\right\}$.

Definition 3.2: Let $\left(F_{A}, \tilde{\tau}\right)$ be a $T_{1}$ soft topological space, then the Vietoris soft topology, denoted by $\tilde{\boldsymbol{\tau}}_{v}$, has the following base:

$\left\langle F_{H_{1}}, F_{H_{z}, \ldots,} F_{H_{n}}\right\rangle=\left\{F_{K} \in \mathcal{C L}\left(F_{A}\right) ; F_{K} \subseteq \bigcup_{\mathrm{t}=1}^{n-1} F_{H_{t}}\right.$ and $F_{H_{t}} \tilde{ก} F_{K} \neq$

$F_{\Phi}$ for each i)

where $F_{H_{1}}, F_{H_{2}, \ldots,} F_{H_{n}}$ are soft open sets in $\left(F_{A}, \tilde{\tau}\right)$.

Example 3.3: Let $U=\left\{u_{1}, u_{2}\right\}, E=A=\left\{x_{1}, x_{2}\right\}$, then $\left(F_{A}, \tilde{\tau}\right)=\left\{F_{\Phi^{\prime}}, F_{A_{1}}, F_{A_{2}}, F_{A_{3}}, F_{A}\right\}$ is a soft topological space, where $\boldsymbol{F}_{A_{1}}=\left\{\left(\boldsymbol{x}_{1},\left\{\boldsymbol{u}_{1}\right\}\right)\right\}, \quad \boldsymbol{F}_{A_{z}}=\left\{\left(\boldsymbol{x}_{2},\left\{\boldsymbol{u}_{2}\right\}\right)\right\}$, $\boldsymbol{F}_{A_{3}}=\left\{\left(x_{1},\left\{u_{1}\right\}\right),\left(x_{2},\left\{u_{2}\right\}\right)\right\}$. Then $\widetilde{\boldsymbol{C}}\left(\boldsymbol{F}_{A}\right)=\left\{\boldsymbol{F}_{A}, \mathbf{F}_{B_{1}}, \mathbf{F}_{B_{z}}, \mathbf{F}_{B_{\mathbf{q}}}\right\}$ where $F_{B_{1}}=\left\{\left(x_{1},\left\{u_{2}\right\}\right)\right\}, \quad F_{B_{z}}=\left\{\left(x_{2},\left\{u_{1}\right\}\right)\right\}$ and $F_{B_{3}}=\left\{\left(x_{1},\left\{u_{2}\right\}\right),\left(x_{2},\left\{u_{1}\right\}\right)\right\}$. So for example, $\left\langle\boldsymbol{F}_{A_{1}}, \boldsymbol{F}_{A}\right\rangle=\left\{\boldsymbol{F}_{A}\right\}$ is a soft open basic set in the soft Vietoris topology defined on $F_{A}$.

Proposition 3.4: (1) $\tilde{\boldsymbol{\tau}}_{\boldsymbol{v}}$ is a $\tilde{T}_{0}$. (2) $\tilde{\boldsymbol{\tau}}_{\boldsymbol{v}}$ is a $\tilde{T}_{1}$.

Proof: (1) Let $F_{K}, F_{H} \in \bar{C}\left(F_{A}\right)$ such that $F_{K} \neq F_{H}$. So without losing generality, there exists $\boldsymbol{\alpha} \tilde{\boldsymbol{\epsilon}} \boldsymbol{F}_{K} \backslash \boldsymbol{F}_{H}$. Now $\left(\boldsymbol{F}_{\boldsymbol{A}} \backslash \boldsymbol{F}_{\mathrm{cx}}\right\rangle$ is an open basic neighbourhood of $\boldsymbol{F}_{H}$ and does not contain $\boldsymbol{F}_{K}$.

(2) Let $F_{K}, F_{H} \approx \mathcal{C} \mathcal{L}_{\left(F_{A}\right)}$ such that $F_{K} \neq F_{H}$ so without losing generality, there exists $\boldsymbol{\alpha} \tilde{\boldsymbol{E}} \mathbf{F}_{\mathrm{K}} \backslash \mathbf{F}_{\mathrm{H}}$. Now $\left\langle\mathbf{F}_{\mathrm{A}} \backslash \mathbf{F}_{\mathrm{x}}\right\rangle$ is an open basic neighbourhood of $\boldsymbol{F}_{H}$ and does not contains $\boldsymbol{F}_{K}$ and $\left\langle\boldsymbol{F}_{\boldsymbol{A}} \backslash \boldsymbol{F}_{H}\right\rangle$ is a basic open neighbourhood of $\boldsymbol{F}_{K}$ and does not contain $\boldsymbol{F}_{H}$.

Lemma 3.5: $\left\langle F_{H_{1},}, F_{H_{2}, \ldots o} F_{H_{n}}\right\rangle=F_{\Phi}$ if there exists at least one $F_{H_{c}}=F_{\Phi}$.

Proof: Suppose $F_{H_{c}}=F_{\Phi}$ for some $\boldsymbol{c} \in\{\mathbf{1}, \mathbf{2}, \ldots, \boldsymbol{n}\}$, then $\left\langle F_{H_{1}}, F_{H_{2}}, \ldots, F_{H_{n}}\right\rangle$

$$
\begin{aligned}
& =\left\{F_{K} \in \bar{C}\left(F_{A}\right): F_{K} \Subset \bigcup_{t=1}^{\bar{n}} F_{H_{t}} \text { and } F_{H_{t}} \text { గF } F_{K} \neq F_{\Phi} \text { for } i\right. \\
& \left.\in\{1,2, \ldots, n\} \backslash\{c\} \text { and } F_{H_{c}} \tilde{F}_{K}=F_{\Phi}\right\}=F_{\Phi} \cdot \text {. }
\end{aligned}
$$


Proposition 3.6: Let $\left(F_{A}, \tilde{\tau}\right)$ be a $\tilde{T}_{1}$ soft topological space, then

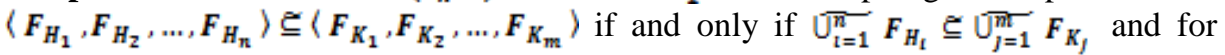
every $\boldsymbol{F}_{K_{j}}$ there exists $\boldsymbol{F}_{H_{i}}$ such that $\boldsymbol{F}_{H_{i}} \subseteq \boldsymbol{F}_{K_{j}}$.

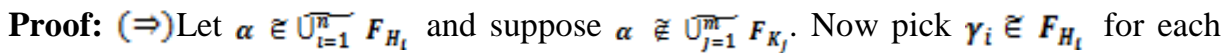
$\boldsymbol{i}=\mathbf{1}, \mathbf{2}, \ldots, n$. Thus each $\boldsymbol{F}_{\gamma_{t}}$ is soft closed subset of $\boldsymbol{F}_{A}$. Hence $\left(\widetilde{U}_{t=1}^{n} \boldsymbol{F}_{\gamma_{t}}\right) \sigma \boldsymbol{F}_{\alpha} \in \overline{\boldsymbol{C}}\left(\boldsymbol{F}_{A}\right)$. It is clear that $\left(\sigma_{t=1}^{n} F_{\gamma_{1}}\right) \sigma F_{\alpha} \notin\left\langle F_{H_{1}}, F_{H_{2}}, \ldots, F_{H_{n}}\right\rangle$. But

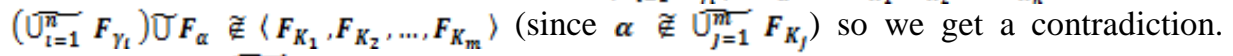
Therefore $\alpha \in \mathrm{U}_{\mathrm{j}=1}^{\mathrm{m}} \boldsymbol{F}_{K_{j}}$.

On the other hand, suppose there is $\boldsymbol{F}_{K_{j}^{*}}$ such that $\boldsymbol{F}_{H_{i}} \nsubseteq \boldsymbol{F}_{K_{j}^{*}}$ for each $\boldsymbol{i}=\mathbf{1}, \mathbf{2}, \ldots, \boldsymbol{n}$.

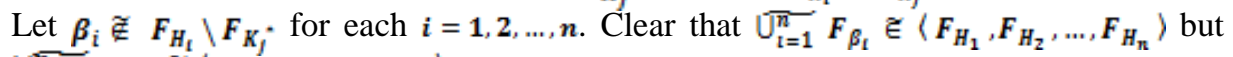
$U_{t=1}^{n=1} F_{\beta_{t}} \widetilde{\notin}\left\langle F_{K_{1}}, F_{K_{z}}, \ldots, F_{K_{m}}\right\rangle$, hence we get a contradiction.

$(\Leftarrow)$ Suppose that $F_{M} \in\left\langle F_{H_{1}}, F_{H_{2}, \ldots,} F_{H_{n}}\right\rangle$, so $F_{M} \subseteq \widetilde{\sigma_{t=1}^{n}} F_{H_{i}}$ and $F_{M} \tilde{F}_{H_{t}} \neq F_{\Phi}$ for each

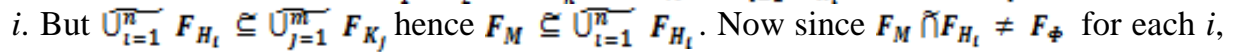
and for each $\mathrm{F}_{\mathrm{K}_{I}}$ there exist $\boldsymbol{F}_{H_{t}}$ such that $\boldsymbol{F}_{H_{t}}$ 巳 $F_{K_{j}}$. Hence $F_{\Phi} \neq F_{M} \tilde{F}_{H_{t}} \subseteq F_{M} \widetilde{F}_{K_{j}}$ for each $j=1,2, \ldots, m$. Therefore $F_{M} \in\left\langle F_{K_{1}}, F_{K_{z}, \ldots,} F_{K_{m}}\right\rangle$.

Proposition 3.7: Let $\left(F_{A}, \tilde{\tau}\right)$ be a $\tilde{T}_{1}$ soft topological space, then $\tilde{\boldsymbol{\tau}}_{v}$ has no soft isolated point if and only if $\boldsymbol{F}_{\mathcal{A}}$ has no soft isolated point.

Proof: $\Leftrightarrow$ ) Supoose $\boldsymbol{F}_{\boldsymbol{A}}$ has a soft isolated point, say $\boldsymbol{\alpha}$, so $\boldsymbol{F}_{\boldsymbol{\alpha}}$ is a soft open in $\boldsymbol{F}_{\boldsymbol{A}}$. Moreover $F_{\alpha}$ is soft closed subset of $\boldsymbol{F}_{A}$ (since $\boldsymbol{F}_{A}$ is $\tilde{T}_{1}$ ). Now, $\left\langle F_{\alpha}\right\rangle=\left\{F_{K} \in \overline{C L}\left(F_{A}\right) ; F_{K} \subseteq F_{\alpha}\right\}=\left\{F_{\alpha}\right\}$. This is a contradiction of being the Vietoris soft topology has no isolated point.

$(\Leftarrow)$ Suppose the Vietoris soft topology has a soft isolated point, say $\boldsymbol{F}_{M}$, so $\left\{\boldsymbol{F}_{M}\right\}$ is a soft open set in $\overline{C L}\left(F_{A}\right)$. Thus there exist $F_{H_{1}}, F_{H_{2}, \ldots o} F_{H_{n}}$ which are soft open in $\boldsymbol{F}_{A}$

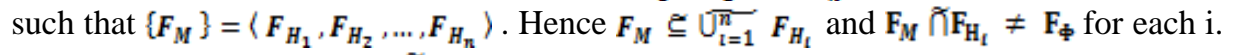
Now, for each $i$ let $\alpha_{i} \notin \mathrm{F}_{M} \bigcap_{\mathrm{F}_{\mathrm{H}_{i}}}$

Fix $F_{\alpha_{l_{e}}}$ for some $\boldsymbol{i}_{*}$ So $\boldsymbol{F}_{\alpha_{\boldsymbol{L}_{s}}}$ soft closed subset of $\boldsymbol{F}_{A}$. Now, $\left\{\boldsymbol{F}_{M}\right\}$ is open in the Vietoris topology, so $\boldsymbol{F}_{\alpha_{L_{\alpha}}}$ is a soft interior point of $\left\{F_{M}\right\}$. Thus there exists a neighbourhood $\left\langle\mathbf{F}_{K_{1},}, \mathbf{F}_{K_{z}, \ldots,}, F_{K_{m}}\right\rangle$ of $F_{\alpha_{L_{s}}}$ such that

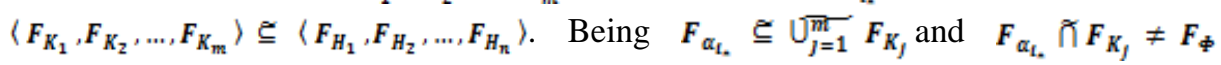
means that $F_{\alpha_{L_{s}}} \tilde{F_{K_{j}}}=\boldsymbol{F}_{\alpha_{\alpha_{s}}}$. Hence $\boldsymbol{F}_{\alpha_{\mathrm{L}_{\mathrm{s}}}}$ is a soft open in $\boldsymbol{F}_{A}$ which is a contradiction of being $\boldsymbol{F}_{A}$ has no soft isolated point.

Proposition 3.8: Let $\left(\boldsymbol{F}_{A}, \tilde{\tau}\right)$ be a soft topological space and let $\left\{\boldsymbol{F}_{K_{n}}\right\}_{n=1}^{\mathrm{w}}$ be any increasing sequence of soft closed sets in the Vietoris soft topology, then $F_{k_{n}} \simeq \mathrm{U}_{n=1}^{\approx} F_{K_{n}}$.

Proof: Let $F_{L}$ be an arbitrary neighbourhood of $U_{n=1}^{m=-1} F_{K_{n}}$ in $\tilde{\tau}_{v}$. Then there exist $F_{H_{1}}, F_{H_{z}, \ldots,} F_{H_{n}}$ soft open in $F_{A}$ such that $\bigcup_{n=1}^{m} F_{K_{n}} \in\left\langle F_{H_{1}}, F_{H_{Z}, \ldots,} F_{H_{m}}\right\rangle \subseteq F_{L}$. Now for every $i \in\left\{\mathbf{1}_{2}, \mathbf{2}_{, \ldots}, \boldsymbol{m}\right\}$, there exist $\boldsymbol{\alpha}_{j} \in\left(\mathrm{U}_{n=1}^{m} F_{K_{n}}\right) \boldsymbol{F}_{H_{j}}$. Thus there exist $\boldsymbol{F}_{K_{n_{j}}}$ such 
that $\boldsymbol{\alpha}_{j} \in F_{K_{n_{j}}}$. Set $\boldsymbol{N}=\max \left\{n_{j} ; \boldsymbol{j}=\mathbf{1}, \mathbf{2}, \ldots, \boldsymbol{m}\right\}$. Then for all $\boldsymbol{j}, \boldsymbol{\alpha}_{j} \in F_{K_{N}}$ and hence for all $j$, $F_{K_{n}} \pi_{F_{H_{j}}} \neq F_{\Phi}$ for all $n \geq N$. Consequently $F_{K_{n}} \in\left\langle F_{H_{1}}, F_{H_{2}, \ldots,} F_{H_{n}}\right\rangle$ for all $n \geq N$.

\section{Conclusion}

This article introduced a hyperspace by depending on the theory of sot closed subsets of a soft topological space. Then this hyperspace is topologised by a certain topology namely, the soft Vietoris topology. Many properties of the new topology are discussed.

\section{Acknowledgment}

The author would like to thank the Iraqi ministry of higher education and scientific research for its support.

\section{References}

1. D. Molodtsov, Comput. Math. Applcat. 37(4/5), 19 (1999). http://dx.doi.org/10.1016/S08981221(99)00056-5

2. M. Shabir and M. Naz, Comput. Math. Applcat. 61, 17 (2011).

3. A. Ibrahim and A. Yusuf, Am. Intern. J. Contemp. Res. 2(9), 205 (2012).

4. L. Vietoris, Monatshefte f ur Mathematik und Physik 32, 258 (1922).

5. E. Michael, Trans. Amer. Math. Soc. 71, 152 (1951). http://dx.doi.org/10.1090/S0002-99471951-0042109-4

6. D. Georgiou and A. Megaritis, Appl. Gen. Topol. 15(1), 93 (2014). http://dx.doi.org/10.4995/agt.2014.2268 